University of Warwick institutional repository: http://go.warwick.ac.uk/wrap

This paper is made available online in accordance with publisher policies.

Please scroll down to view the document itself. Please refer to the repository record for this item and our policy information available from the repository home page for further information.

To see the final version of this paper please visit the publisher's website. Access to the published version may require a subscription.

Author(s): Amrita Dhillon \& Carla Perroni (Warwick)

Article Title: Tax Earmarking and Grass roots Accountability

Year of publication: 2001

Link to published version:

http://www.sciencedirect.com/science/article/B6V84-430G2HM-

$\mathrm{H} / 2 / 3546 f 2 b 5 f 609839 a d 298 f 28 f 97666758$ 


\title{
Tax Earmarking and Grass-roots Accountability
}

\author{
Amrita Dhillon and Carlo Perroni \\ University of Warwick
}

July 2000

\begin{abstract}
We ask whether tax earmarking can foster accountability in public provision of goods and services when consumers can privately monitor provision. We show that earmarking can raise the stakes that consumers have in monitoring public provision independently of how taxes are earmarked, because it introduces a more direct linkage between monitoring and taxes paid.
\end{abstract}

KEY WORDS: Monitoring, Incomplete Information, Accountability.

JEL CLASSIFICATION: H1, H7, D8

Correspondence should be addressed to Amrita Dhillon, Department of Economics, University of Warwick, Coventry CV4 7AL, UK. E-mail: A.Dhillon @warwick.ac.uk. 


\section{Introduction}

This note examines how tax earmarking affects the extent to which spending authorities are accountable to taxpayers. Earlier literature on fiscal accountability has typically adopted a top-down view of accountability, where spending agents are monitored by some higher-ranking institution like a regulatory authority. Here we focus instead on a bottom-up notion of accountability, which we could label 'grass-roots accountability': monitoring of spending authorities by the final users of the publicly provided goods.

Buchanan's (1963) early contribution to the analysis of tax earmarking focused on its implications for fiscal choices under majority voting. Although later authors have studied different aspects of tax earmarking, a potentially important dimension of it has so far been neglected, namely, the linkage between tax earmarking and private monitoring of spending agencies by taxpayers. The idea that private agents' involvement can play an important role in ensuring fiscal discipline has been a recurring theme in the debate over government provision of services both in the Europe and North-America, although it has received comparatively less attention in the theoretical literature; the only paper we know of to explicitly look at this issue is Davis and Hayes (1993). In what follows, we explicitly formalize this idea and explore its implications for tax earmarking.

We describe a model of fiscal choices where the spending agencies that are responsible for the provision of public goods and services have private information about the costs they incur, and where citizens can undertake costly monitoring whenever they receive goods and services from the agency. We then characterize mixed-strategy equilibria of the resulting noncooperative game and examine how they depend on the tax earmarking regime.

Our findings show that earmarking certain taxes to certain uses can foster accountability of spending authorities to taxpayers independently of the manner in which taxes and spending categories are matched. This is because, as long as private agents make differential contributions to different revenue sources (because of preference or endowment heterogeneity), earmarking can reduce free-riding in private monitoring. ${ }^{1}$

The structure of the paper is as follows. Section 2 describes the model. Section 3 considers the no-earmarking case, and Section 4 that of full earmarking. Section 5 compares the two and concludes.

\section{The Model}

We model the effects of earmarked taxes by considering two agencies, denoted $A$ and $B$, providing

\footnotetext{
${ }^{1}$ It is well understood that institution design can help reduce free riding in private monitoring. Laffont and Tirole (1994, Chapter 15) discuss this idea in a model of regulatory capture, and show that the optimal pricing rule to be adopted by the regulator may depend on whether or not the regulator is benevolent: with a self-interested regulator, Average Cost Pricing regulation may outperform Marginal Cost Pricing regulation because it encourages consumers to monitor the regulator. Our analysis can be viewed as an application of these ideas to the case of self-interested government agencies providing public goods, with earmarking rules playing a role analogous to that played by pricing rules in a regulatory context.
} 
two different public goods, to $2 n$ consumers. Thus, good $i \in\{A, B\}$ is produced by agency $i$, combining a certain amount of labour inputs, $e$ (effort by the agency), with other intermediate inputs, $r$, according to technologies which can be represented by a concave production function $q\left(e, r, \theta_{i}\right)$, where $\theta_{i}$ represents a factor-neutral shift parameter. The possible input combinations needed to produce a unit of the good $A$ can be represented by a unit isoquant described by the condition $q\left(e, r, \theta_{i}\right)=1$. For the purposes of our analysis, it is convenient to represent substitution possibilities between the two inputs by means of functions $e\left(r, \theta_{i}\right)$ and $r\left(e, \theta_{i}\right)$, both increasing in $\theta_{i}$ and decreasing respectively in $r$ and $e$. Technologies are subject to random exogenous shocks which are modelled as shifts in $\theta_{i}$, and are assumed to be independent and uncorrelated across agencies. We assume that there are only two possible realizations: $\underline{\theta}$ and $\bar{\theta}$, with $\bar{\theta}>\underline{\theta}$, occurring with probabilities respectively equal to $\underline{\pi}$ and $\bar{\pi}=1-\underline{\pi}$ (the same possible lotteries on outcomes are assumed for both agencies) .

Each agency is viewed as being composed of bureaucrats who are paid a fixed salary $W$ to exert an effort equal to unity, and who have private information about cost conditions. If an agency reveals the true state of technologies, it will be required to exert an effort equal to one, which involves using an amount $r\left(1, \theta_{i}\right)$ of inputs, with $i \in\{A, B\}$. If agency $i$ misrepresents the true technological conditions when $\theta_{i}=\underline{\theta}$, it will be able to employ an amount of inputs equal to $r(1, \bar{\theta})$ and reduce its effort to $e(r(1, \bar{\theta}), \underline{\theta})<e(r(1, \underline{\theta}), \underline{\theta})=1$, while if the misrepresentation occurs when $\theta_{i}=\bar{\theta}$, effort will increase to $e(r(\underline{\theta}), \bar{\theta})>e(r(\bar{\theta}), \bar{\theta})=1$. The cost of the publicly provided good thus depends on the realization of the state and on whether or not the agency "lies". In the following, we shall denote by $\lambda_{i}\left(\theta_{i}\right) \in\{0,1\}$ the agency's reporting strategy, with $\lambda\left(\theta_{i}\right)=0$ if the agency tells the truth and $\lambda_{i}\left(\theta_{i}\right)=1$ if it lies. We shall also make use of the shorthand $R\left(\theta_{i}\right) \equiv r\left(1, \theta_{i}\right)$.

Although information about costs is private, individual citizens can undertake costly monitoring of the local agencies effort whenever they receive goods and services. The idea here is that the public have an opportunity to directly observe bureaucratic performance and have channels that they can use to censor abuses (such as formal complaints or other forms of legal or politically relevant actions). If any of the citizens decides to monitor the agency and the agency has lied about cost conditions, the true state will be uncovered with certainty, and the agency will be forced to exert an effort equal to 1 and incur a penalty $F$.

Let us denote by $\mu_{h}^{i} \in\{0,1\}, h \in\{1, \ldots, 2 n\}$, citizen $h$ 's monitoring choice, with $\mu_{h}^{i}=1$ representing the choice to monitor agency $i$ by a citizen $h$ and $\mu_{h}^{i}=0$ the choice not to monitor; also, let $\vec{\mu}^{i}$ denote the profile of consumers' monitoring choices with respect to agency $i$. Then, the cost of public good provision by agency $i$, denoted as $c_{i}$, depends on technological conditions and on the strategies of both the agency and the citizens:

$$
\begin{array}{r}
c_{i}\left(\theta_{i}, \lambda_{i}\left(\theta_{i}\right), \vec{\mu}^{A}\right) \equiv W+\left(1-\left(1-\Gamma^{i}\left(\vec{\mu}^{i}\right)\right) \lambda_{i}\left(\theta_{i}\right)\right) R\left(\theta_{i}\right)+\left(1-\Gamma^{i}\left(\vec{\mu}^{i}\right)\right) \lambda_{i}\left(\theta_{i}\right) R\left(\hat{\theta}_{i}\right), \\
i \in\{A, B\},
\end{array}
$$

where $\Gamma^{i}\left(\vec{\mu}^{i}\right) \equiv \mathbb{I}_{\sum_{h} \mu_{h}^{i}>0}$ is an index function which takes a value of 1 whenever $\sum_{h} \mu_{h}^{i}>0$, i.e. when at least one citizen monitors, and is zero otherwise. 
Monitoring is costly for consumers. Consumers are endowed with a certain amount of time which they can either sell on the labour market at a given wage rate or use for monitoring an agency (or both agencies). We shall denote with $Y$ potential before-tax income, and with $M$ the opportunity cost (in terms of foregone earnings) of monitoring one agency. The monitoring cost increases to $2 M$ if the consumer decides to monitor both agencies.

The agencies' services are paid for by consumer taxes, which may or may not be earmarked to a specific agency. Taxes are anonymous, but can affect different individuals differentially because of their different consumption patterns: a tax on cigarettes is only paid by smokers (if one abstracts from general equilibrium effects) and a tax on fuel is only paid by car users. Here, we shall make the extreme assumption that there exist two separate consumer types, 1 and 2, each consisting of $n$ individuals, each buying a private good - completely unrelated to the public goods supplied by the agencies - in a fixed amount equal to unity: type- 1 consumers consume private good 1 on which a unit tax at rate $t_{1}$ is levied, and type- 2 consumers consume private good 2 and pay a unit tax $t_{2}{ }^{2}$

If tax revenues are not earmarked, then the total cost of providing the public goods is financed by the sum of the revenues from each tax. If we let $t_{1}=t_{2} \equiv t$, then the tax required will be

$$
t_{1}^{N E}\left(c_{A}, c_{B}\right)=t_{2}^{N E}\left(c_{A}, c_{B}\right)=t^{N E}\left(c_{A}, c_{B}\right)=\frac{c_{A}+c_{B}}{2 n} .
$$

Earmarking of taxes can be achieved by instituting a link between a specific tax revenue flow and a certain agency. Suppose, arbitrarily, that the cost of provision incurred by agency $A$ is financed by taxes on good 1 , with the cost for $B$ being financed by taxes on good 2 . Then we have

$$
t_{1}^{E}\left(c_{A}, c_{B}\right)=\frac{c_{A}}{n} ; \quad t_{2}^{E}\left(c_{A}, c_{B}\right)=\frac{c_{B}}{n} .^{3}
$$

Denote the payoff of a representative type- $j(j \in\{1,2\})$ consumer $k \in\{1, \ldots, n\}$ as $Z_{k}^{j}$. Then,

$$
\begin{aligned}
& Z_{k}^{j}\left(\theta_{A}, \theta_{B} ; \lambda_{A}\left(\theta_{A}\right), \lambda_{B}\left(\theta_{B}\right) ; \mu_{k}^{A}, \mu_{k}^{B} ; \vec{\mu}_{-k}^{A}, \vec{\mu}_{-k}^{B}\right) \equiv \\
& \quad U\left(Y-\mu_{k}^{A} M-\mu_{k}^{B} M-t_{j}\left(c_{A}\left(\theta_{A}, \lambda_{A}\left(\theta_{A}\right), \vec{\mu}^{A}\right), c_{B}\left(\theta_{B}, \lambda_{B}\left(\theta_{B}\right), \vec{\mu}^{B}\right)\right)\right), \quad j \in\{1,2\},
\end{aligned}
$$

where $\vec{\mu}_{-h}^{i}, i \in\{A, B\}$ are the monitoring choices of all consumers except $h$, and $U$ denotes utility. Note that in this formulation citizens do not directly gain from the application of the penalty if misrepresentation by the agency is discovered: the benefit of monitoring for the citizens only consists of potential cost savings which result in lower taxes. Throughout our analysis we shall assume risk-neutral citizens, i.e. $U(Y)=Y{ }^{4}$

\footnotetext{
${ }^{2}$ There is no reason to restrict the number of consumer types to two, to assume that each consumer type is only affected by a single tax (typically individuals are affected by all taxes), or to assume consumption to be independent of level of taxation, except that this simplified specification is convenient and sufficient to develop our argument. Clearly, tax earmarking raises distributional considerations, which are not captured by our symmetric treatment of consumers and which would come into play in a more realistic model.

${ }^{4}$ Adopting a more general utility function allowing for risk aversion would introduce a tradeoff between insurance and monitoring incentives, which is not essential to our argument.
} 
Each agency's payoff only depends on the state and strategies directly affecting it, and can be expressed as

$$
\begin{aligned}
& L_{i}\left(\theta_{i}, \lambda_{i}\left(\theta_{i}\right), \vec{\mu}^{i}\right) \equiv \\
& \quad W-\left(1-\left(1-\Gamma^{i}\left(\vec{\mu}^{i}\right)\right) \lambda_{i}\left(\theta_{i}\right)\left(1-e\left(R\left(\hat{\theta}_{i}\right), \theta_{i}\right)\right)\right) v-\Gamma^{i}\left(\vec{\mu}^{i}\right) \lambda_{i}\left(\theta_{i}\right) F, \quad i \in\{A, B\},
\end{aligned}
$$

where $v$ the constant marginal valuation of effort. For the agency, misrepresenting cost conditions involves a change in effort and the prospect a penalty $F$ if monitoring occurs. ${ }^{5}$ In the unfavourable state $(\theta=\bar{\theta})$, telling the truth will always be a dominant strategy for the agency, since it results in lower effort and no penalty; hence in the remainder of our discussion we shall take $\lambda(\bar{\theta})=0$. But, in the favourable state $(\theta=\underline{\theta})$, the agency will have an incentive to misrepresent cost conditions in order to reduce effort, which it will have to weigh against the potential penalty it faces if monitoring takes place.

The citizens and the agencies play an 'inspection game' (Laffont and Tirole, 1994) where the agencies announce their cost conditions and, simultaneously ${ }^{6}$ consumers decide whether or not to monitor the agencies. In this game, each agency can adopt a mixed strategy, whereby it cheats (misrepresent cost) with probability $q_{i}$ and does not cheat with probability $1-q_{i}$. Each citizen can similarly mix between monitoring and not monitoring either or both agencies. The monitoring probabilities of the citizens are denoted as $p_{h}^{i}$, with $i \in\{A, B\}$, and $h \in\{1, \ldots, 2 n\}$. Pure- or fully mixed-strategy equilibria can arise in this game, depending on parameter values.

\section{No Earmarking}

We shall begin by considering first the no-earmarking case. A consumer has four possible actions, to monitor one of $A$ or $B$, monitor both agencies, or monitor neither. Note that there will never be pure-strategy equilibria where any of the citizens monitors an agency with probability one: if monitored with certainty, an agency would choose never to cheat, which in turn implies that monitoring all the time could not be an equilibrium best response. Hence, the only possible purestrategy equilibrium involves all citizens choosing never to monitor and both agencies cheating:

\footnotetext{
${ }^{5}$ For expositional convenience we assume that the penalty is incurred whenever the agency lies, whatever the state. It may seem incongruous to punish the agency for exerting extra effort when $\theta=\bar{\theta}$; as we shall see, however, the agency never lies in this case, independently of whether or not a penalty is applied, and therefore this assumption is fully innocuous.

${ }^{6}$ We model the game as simultaneous rather than sequential for simplicity. They key feature here is that monitoring by citizens encourages agencies to be more efficient. Sequentiality would not add much to this basic incentive structure, other than the ability by consumers to compare announcements across different agencies and update their priors accordingly.
} 
Proposition 1: There exists a (symmetric) pure-strategy Nash equilibrium which involves all citizens choosing not to monitor and all agencies cheating iff

$$
M>\frac{\pi}{2 n}(\bar{\theta}-\underline{\theta}) .
$$

Proof: Consider consumer $h$. Given $p_{s}^{i}=0, \forall s \neq h, i \in\{A, B\}$, and $q_{A}=q_{B}=1$, consumer $h$ would choose not to monitor, given that no other consumer monitors, if and only if the expected utility from monitoring an agency-which equals $Y-M-((1+\bar{\pi}) \bar{\theta}+\underline{\pi \theta}) /(2 n)$-is less than that from not monitoring it-which equals $Y-\bar{\theta} / n$; this implies condition (6) above.

Such a pure-strategy equilibrium will only prevail in situations where monitoring costs are too high from the point of view of individual consumers for monitoring to be worthwhile, and is therefore not very interesting. Note that, for a given $M$, the right-hand side of (6) is decreasing in $n$, which implies that private monitoring can only be observed if group size is not "too large", and is therefore more relevant for situations where the number of taxpayers is relatively small, as in the case of local agencies providing goods paid for by local taxes.

If $M<\underline{\pi}(\bar{\theta}-\underline{\theta}) /(2 n)$ and there are no symmetric pure-strategy equilibria, by the Nash existence theorem there will exist a symmetric-mixed strategy (Bayesian) equilibrium in which the consumers randomize between inspecting and not inspecting and the agency always announces the truth when $\theta=\bar{\theta}$ and lies with some probability when $\theta=\underline{\theta}$. Let

$$
\Omega \equiv \frac{(1-\alpha) v}{F+(1-\alpha) v}<1 .
$$

Then, we can characterize symmetric mixed-strategy equilibria as follows:

Proposition 2: A mixed-strategy equilibrium with no earmarking has

$$
p_{h}^{A}=p_{h}^{B}=p^{* N E}=1-(1-\Omega)^{1 /(2 n)},
$$

and

$$
q_{A}=q_{B}=q^{* N E}=\frac{2 M(1-\Omega)^{1 /(2 n)}}{\underline{\pi}(1-\Omega)(\bar{\theta}-\underline{\theta})}
$$

Proof: In a fully-mixed equilibrium, each agency, when facing a favourable cost realization, must be indifferent between telling the truth and lying. Let $\vec{p}$ represent the profile of consumers' mixed strategies, and $\vec{p}_{-h}$ the vector of mixing probabilities of all citizens except $h$. When $\theta=\underline{\theta}$, the expected payoff for an agency $i$ as a function of her reporting strategy $\lambda_{i}(\underline{\theta})$ is given by the expectation $E\left(L_{i}\left(\underline{\theta}, \lambda_{i}(\underline{\theta}), \vec{\mu}^{i}\right) \mid \vec{p}\right) \equiv \Phi\left(\lambda_{i}(\underline{\theta}), \vec{p}\right)$. Indifference between truthfully reporting costs and lying requires

$$
\Phi(1, \vec{p})=\Phi(0, \vec{p}), \quad i \in\{A, B\} .
$$

Because of risk neutrality and of the fact that monitoring costs are linear in the number of agencies monitored, the expected payoff from any strategy combination $\left(\mu_{h}^{A}, \mu_{h}^{B}\right)$ to any citizen 
$h$ is additively separable across agencies. Thus, in a symmetric mixed-strategy equilibrium we can simply require that a consumer be indifferent between never monitoring a certain agency $i$ and monitoring it with probability one, given the strategies of all other players. Suppose then that a consumer monitors $A$ with probability $p_{h}^{A}$. Consumer $h$ 's expected payoff as a function of her monitoring choice $\mu_{h}^{B}$ with respect to agency $B$ is then given by the expectation $E\left(Z_{h}\left(\theta_{A}, \theta_{B} ; \lambda_{A}\left(\theta_{A}\right), \lambda_{B}\left(\theta_{B}\right) ; \mu_{h}^{A}, \mu_{h}^{B} ; \vec{\mu}_{-h}^{A}, \vec{\mu}_{-h}^{B}\right) \mid \bar{\pi}, \underline{\pi}, q_{A}, q_{B}, \vec{p}_{-h}, p_{h}^{A}\right) \equiv \Psi\left(\mu_{h}^{B} ; q_{A}, q_{B}, \vec{p}_{-h}, p_{h}^{A}\right)$. Indifference requires

$$
\Psi\left(1 ; q_{A}, q_{B}, \vec{p}_{-h}, p_{h}^{A}\right)=\Psi\left(0 ; q_{A}, q_{B}, \vec{p}_{-h}, p_{h}^{A}\right), \quad h \in\{1, \ldots, 2 n\} .
$$

Substituting expressions (4), (5), and (2) into (10) and (11), and letting $q_{i}=q^{* N E}, i \in\{A, B\}$, and $p_{h}^{i}=p^{* N E}, h \in\{1, \ldots, 2 n\}, i \in\{A, B\}$, gives conditions (8) and (9) above.

Note that the equilibrium probability of monitoring is directly related to the savings in effort from cheating-represented by $(1-\alpha) v$ in $\Omega$-and inversely related to the penalty. The aggregate monitoring effort, as measured by the probability of being monitored for an agency - which equals $1-\left(1-p^{* N E}\right)^{2 n}=(1-\alpha) v /(F+(1-\alpha) v)$-is independent of the number of consumers involved. The equilibrium probability of cheating, on the other hand, is inversely related to the number of individuals (the denominator of (9) is decreasing in $n$ ). Thus, once the conditions for a mixedstrategy equilibrium are met, free-riding incentives in monitoring are reflected in the equilibrium level of cheating.

\section{Full Earmarking}

With full earmarking, the taxes payable by a consumer only depend on the action of the agency to which the revenues from such taxes are earmarked. Consider, for example, a consumer $k \in$ $\{1, \ldots, n\}$ of type 1 whose taxes are earmarked to agency $A$. The choice to monitor both agencies is clearly dominated by that of monitoring agency $A$ only. The agencies thus face the same payoffs as in the no earmarking case, except that now the number of consumers that can potentially monitor any agency is now only $n$.

As in the no-earmarking case, we can determine an upper bound on $M$ above which consumers will never choose to monitor (the proof is analogous to that of Proposition 1):

Proposition 3: Under full tax earmarking, there exists a (symmetric) pure-strategy Nash equilibrium which involves all citizens choosing not to monitor and all agencies cheating iff

$$
M>\frac{\pi}{n}(\bar{\theta}-\underline{\theta}) .
$$

Note that (12) implies (6). Thus, if there is no symmetric pure-strategy equilibrium in the full earmarking case, then there is not one in the no earmarking case either. Symmetric mixed-strategy equilibria under full earmarking can be characterized as follows: 
Proposition 4: When taxes are fully earmarked, the equilibrium probability of monitoring and of cheating in the symmetric mixed-strategy equilibrium is given by

$$
p_{k}^{1 A}=p_{k}^{2 B}=p^{* E}=1-(1-\Omega)^{1 / n} .
$$

and

$$
q_{A}=q_{B}=q^{* E}=\frac{M(1-\Omega)^{1 / n}}{\underline{\pi}(1-\Omega)(\bar{\theta}-\underline{\theta})} .
$$

PROOF: With full earmarking there are only two possible meaningful actions possible for a consumer: she can either monitor the agency to which the taxes she pays are earmarked or not monitor at all. Suppose that, arbitrarily, the revenues from taxes paid by type- 1 consumers are earmarked to agency $A$, with the same applying to type-2 consumers and agency $B$, and focus on the monitoring choice of type-1 consumers. The expected payoff for a representative consumer $k \in\{1, \ldots, n\}$ of type 1 as a function of her monitoring choice with respect to agency $A$, and given that no type- 1 consumer will never monitor $B$, is given by the expectation $E\left(Z_{k}^{1}\left(\theta_{A}, \theta_{B} ; \lambda_{A}\left(\theta_{A}\right), \lambda_{B}\left(\theta_{B}\right) ; \mu_{k}^{A}, 0 ; \vec{\mu}_{-k}^{A}, \vec{\mu}_{-k}^{B}\right) \mid\right.$ $\left.\bar{\pi}, \underline{\pi}, q_{A}, q_{B},\left(\vec{p}_{-k}^{1 A}, \overrightarrow{0}\right),\left(\overrightarrow{0}, \vec{p}_{-k}^{2 B}\right)\right) \equiv \hat{\Psi}^{1}\left(\mu_{k}^{1 A} ; q_{A}, q_{B},\left(\vec{p}_{-k}^{1 A}, \overrightarrow{0}\right),\left(\overrightarrow{0}, \vec{p}_{-k}^{2 B}\right)\right)$. Indifference requires

$$
\hat{\Psi}^{1}\left(1 ; q_{A}, q_{B}, \vec{p}_{-h}\right)=\hat{\Psi}^{1}\left(0 ; q_{A}, q_{B}, \vec{p}_{-h}\right), \quad k \in\{1, \ldots, 2 n\} .
$$

The same applies to type-2 consumers with a change of indices.

Using (15) in conjunction with (10), substituting expressions (4), (5), and (3) into (10) and (15), and letting $q_{i}=q^{* E}, i \in\{A, B\}$, and $p_{k}^{1 A}=p_{k}^{2 B}=p^{* E}, k \in\{1, \ldots, n\}$, gives conditions (13) and (14) above.

\section{Comparison and Conclusions}

We will now show that under our assumptions there will be an unambiguous decrease in cheating when earmarking is used:

Proposition 5: Assume $M<\underline{\pi}(\bar{\theta}-\underline{\theta}) / n$. Then, the equilibrium probability of cheating by a representative agency is less under full tax earmarking than with no earmarking, i.e. $q^{* E}<q^{* N E}$.

Proof: Comparing expressions (14) and (9), it is easily seen that $q^{* N E}>q^{* E}$ requires $2(1-\Omega)^{1 / 2 n}>$ $(1-\Omega)^{1 / n}$. Since $(1-\Omega) \in(0,1)$, this condition is always met.

Tax earmarking thus provides a way of mitigating agency problems in public provision. Moreover, it should be stressed that the precise manner in which taxes are earmarked is totally immaterial for it to be effective: in the model just described the tax base here is completely independent of the goods and services publicly provided, and the assignment of tax bases to spending categories is fully arbitrary. This means that, even when user charges are not available - as is the case with health and education services in many countries - tax earmarking can still play a positive role in fostering accountability, particularly with respect to local government agencies. 


\section{References}

Buchanan, J.M. (1963) "The Economics of Earmarked Taxation," Journal of Political Economy $71,457-469$.

Davis, M.L., and K. Hayes (1993) "The Demand for Good Government," Review of Economics and Statistics 75, 148-152.

Laffont, J.J., and J. Tirole (1994) A Theory of Incentives in Procurement and Regulation, MIT Press, Cambridge, Ma. 